\title{
Geometrical Design and Operability Verification of a Proportional Pressure Relief Valve
}

\author{
M. Erhard, J. Weber, and G. Schoppel* \\ Institut für Fluidtechnik, Technische Universität Dresden, Germany \\ E-mail: erhard@ifd.mw.tu-dresden.de,weber@ifd.mw.tu-dresden.de, georg.schoppel@boschrexroth.de \\ *Bosch Rexroth AG, Germany
}

\begin{abstract}
This paper demonstrates a novel numerical procedure for a synthesis of electro-hydraulic pressure relief valve performance starting with the performance requirements from a functional specification. Results of this methodological approach are the unknown demands for the solenoid with regard to control orifice concepts and the solenoid geometry itself fulfilling the requirements.

The developed modeling approach comprises two major solution steps. At the beginning the displacement-dependent solenoid-spring force characteristic is calculated from the intended pressure-flow rate characteristic map without the use of optimization tools. This new algorithm performs an inverse simulation of a single stage pressure valve. The next solution step deals with the determination of the geometrical shape of the solenoid. Here steady state FEM computations are used in combination with response surface methodologies to predict the desired geometrical shape. At the end robustness verification is done by means of Monte-Carlo simulation for the uncertainties of the valve assembly.

Starting point for the inverse calculations are different control orifice concepts causing varying demands on the solenoid. As a result of this benchmark the most favorable control orifice concept is used for determination of the solenoid geometry. This is performed within several iterations also considering the spring force. Afterwards the robustness of the valve assembly is examined. The verification of the modeling attempt is done on the basis of prototype parts for the solenoid and the control orifice. Measurements confirm the accuracy of the novel simulation strategy for a standalone virtual product development.
\end{abstract}

Keywords: pressure relief valve, inverse simulation model, solenoid, CFD, FEM

\section{Introduction}

Nowadays, simulation techniques in virtual product development are more and more efficient and reliable offering the possibility for the prediction of valve performance by using only simulation methods. The different simulation techniques cover a wide range of applications, whereby the temporal and spatial distribution of the inner field variables as well as the overall system behavior is in the focus of the investigations. The acquired transparency of the inner field variables in combination with the resulting component performance establishes a basis for a knowledge-based further development of these electro-hydraulic components.

Nevertheless, the complex and nonlinear character of electro-hydraulic valves and its physical-based description were already part of previous scientific work not all fields of application are considered until now. In particular, this includes without limitation the prediction of the geometrical shape of the different physical subsystems of an electro- hydraulic valve outlined in fig. 1 with respect to an intended and predefined valve performance, respectively. Although modern simulation techniques, e.g. finite-element-methods (FEM) or computational fluid dynamics (CFD), establish a relation between the geometrical shape and the subsystem performance including solenoid force, pressure drop or pressure distribution, in general no explicit formulation of these linkages is possible.

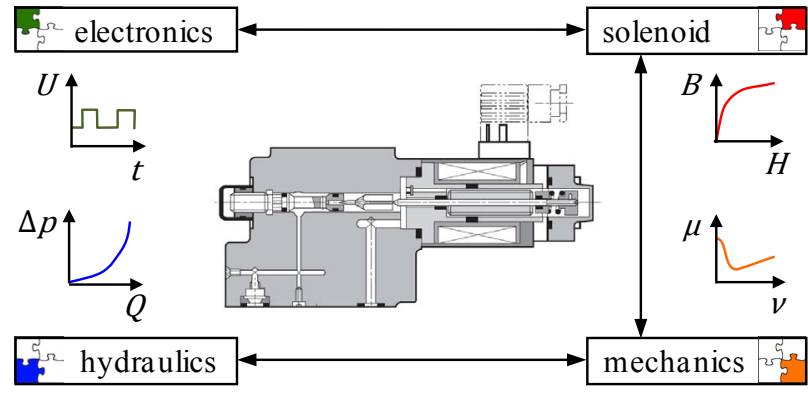

Figure 1: Physical subsystems of electro-hydraulic valves 
This describes the well known problem of inverse simulation techniques. Normally, the so called "forward simulation" or "analysis" gives a suitable solution whereas the "inverse simulation" or "synthesis" requires particular solution strategies. Leaving the subsystem level, see fig. 2, the same problem exists on the component level. Starting from an intended valve performance the unknown subsystem characteristics have to be determined initially followed by the estimation of an appropriate geometrical shape.

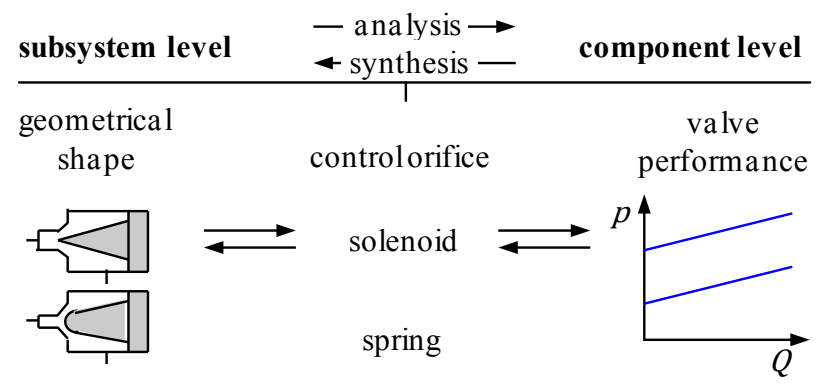

Figure 2: Simulation inputs and outputs (schematic)

As outlined above, the simulation techniques presented in literature predominantly focus on the analysis of existing systems, the verification of the methodological approach or the failure analysis. The mathematical analysis of a directoperated pressure valve $[1,2]$ reveals fundamental relations, especially concerning the dynamic valve behavior. Because no detailed parameters for the flow region are taken into consideration, no linkage between the geometrical shape and the valve performance can be established. An extension of this initial analysis approach is given through the involvement of detailed field simulation techniques for parameter extraction, namely FEM and CFD. Current papers [3] illustrate the modeling approach for electro-hydraulic proportional valves using FEM for parameterization of the solenoid and simplified parameters for the hydraulic and mechanical subsystem. Other authors $[4,5]$ use CFD calculations for the hydraulic part of the valve, gaining a deeper insight of the internal flow and the force on the valve spool. This enables more detailed simulation models and broadens its range of application such as a failure analysis based on the geometrical tolerances of the flow region. A combination of both modeling strategies is presented in [6] predicting the whole component performance without measurements using a holistic approach. Recent developments [7, 8] merge the advantage of a detailed flow simulation with the possibility of component performance predictions. These so called fluid-structure interaction simulations create new opportunities for the investigation of significant more complex problems.

However, there is a similarity between these modeling approaches: each focuses on the analysis of an existing system. The more interesting issue primarily in industrial applications deals with the inverse problem. Here, the functional specification defines the valve performance and the necessary subsystem characteristics as well as the associated geometrical shapes are unknown. Usually, this is done by a trial\&error process but simulation techniques become more and more important in this field of application. The imple- mentation of these techniques on the component level for the hydraulic subsystem is presented in [9] optimizing the relief valve performance based on a holistic simulation approach. Because no linkage between the geometrical shape of the flow region and the describing parameters exist radical changes of the flow region aren't permissible.

This paper takes up this gap by establishing an inverse simulation approach for a direct-operated proportional pressure relief valve. An initial approach for this idea was already presented by the authors in a preceding paper [10]. The inverse simulation is carried out in two major steps starting on the component level with the calculation of the unknown subsystem characteristics. Afterwards, the geometrical shape fulfilling the subsystem requirements is predicted. Because of the multi-dimensionality of this task a simplification step is introduced. The problem dimension is reduced by replacing the degrees of freedom of the control orifice in a way that predefined concepts are investigated.

The simulation model developed by the authors demonstrates the conceptual and geometrical design of a pressure valve starting from the intended valve performance. Since the expected valve performance is predicted only using nominal values of the influencing parameters a further robustness analysis is implemented. The additional information facilitates the interpretation of the prototype performance. Final measurements of solenoid and valve characteristics verify the quantitatively accurate predictions and show the potentials of this novel methodology.

\section{Proportional pressure relief valves and its modeling}

The component analyzed in this work is a direct-operated proportional pressure relief valve with its main elements illustrated in fig. 3. Increasing demands in power-density are the driving factors for new solutions for these valves, whereby further enhancement in valve performance is restricted through the limits determined by physical laws. Decreasing component sizes simultaneously linked with increasing nominal flow rates result in higher energy losses and actuator forces. In conjunction with minimized electric power consumption valve operability strongly depends on highly-adapted flow conditions and actuator concepts. Pressure relief valves are generally used for pressure limitation or pressure setting, whereas the pressure-flow rate characteristic curve is a crucial property. Additionally, the dynamic behavior has an important role especially the stability in all operating points. Dynamic analyzes of this valve aren't part of this paper, neglecting all derivatives with respect to time.

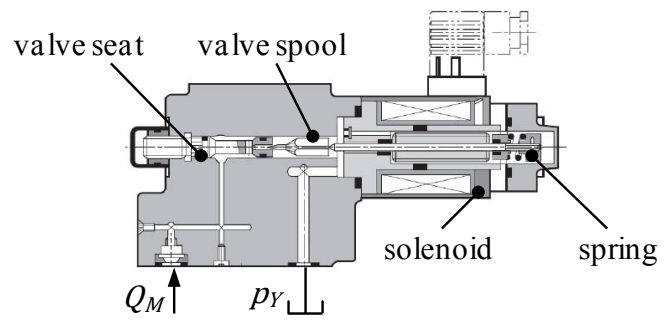

Figure 3: Assembly structure of investigated pressure valve 
Electro-hydraulic valves, just as the proportional pressure relief valve outlined in fig. 3 are characterized through complex interactions between the different physical subsystems. Special emphasis should be placed on the influence of the valve seat/valve spool combination in conjunction with the solenoid-spring force characteristics on the system performance. Therefore, a well-coordinated geometrical shape of both subsystems offers the possibility for great performance enhancements. Hence, knowledge and transparency of the spatial distribution of the inner field variables as well as understanding the major interactions between these different subsystems are essentially for a successful valve design.

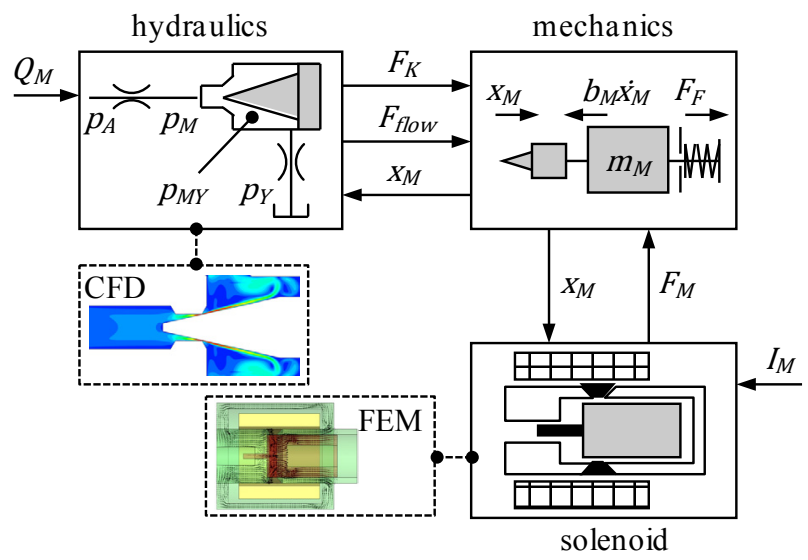

Figure 4: Modeling structure

The modeling structure, see fig. 4 is directly derived from the element structure of the valve assembly; the electronics is not part of the investigations. A detailed description of the remaining subsystems is outlined in the following subsections.

\subsection{Hydraulic subsystem}

Irreversible energy losses and the force reaction on the valve spool are considered in this subsystem. In case of pressure valves these losses occur predominantly at the control orifice, additional losses are in the upstream and downstream flow region. The loss coefficient $\zeta$ for hydraulic resistances with cross-section $A_{\text {flow }}$ is calculated with eq. (1). Therein $\Delta p$ represents the pressure loss over a given system length with closest cross-section $A_{\text {flow }}$ at flow rate $Q_{M}$. For more complicated control orifice designs the application of an alternative modeling approach in form of characteristic maps following eq. (2) is also conceivable.

$$
\begin{gathered}
\zeta=\frac{\Delta p}{\frac{\rho}{2}\left(\frac{Q_{M}}{A_{\text {flow }}}\right)^{2}} \\
\Delta p_{M-M Y}=\mathrm{f}\left(Q_{M}, x_{M}\right)
\end{gathered}
$$

With the knowledge of pressure losses the static pressures $p_{A} / p_{M} / p_{M Y}$ in the valve as well as the force reactions on single boundaries of the flow region can be calculated. Here, the valve spool is the element of interest. The static pressure on the valve spool decreases continuously once the fluid is accelerated in proximity to the control orifice, see fig. 5 . The resulting valve spool force is influenced through the appearing pressure profile making and adjustment of the force equation necessary. Therefore, eq. (3) concerning the force reaction $F_{\text {spool }}$ is split into a theoretical pressure force $F_{K}$ and a corrective term $F_{\text {flow }}$ called flow force. The model eq. (4) for the flow force is determined by conservation of momentum, whereas $k_{G F}$ represents a preliminary unknown coefficient characterizing the control orifice design.

$$
\begin{gathered}
F_{\text {spool }}=F_{K}-F_{\text {flow }} \\
F_{\text {flow }}=\frac{\rho Q_{M}^{2}}{A_{\text {flow }}} k_{G F} \\
F_{\text {flow }}=\mathrm{f}\left(Q_{M}, \Delta p_{M-M Y}\right)
\end{gathered}
$$

If the results for the geometrical coefficient show no clear allocation, an adequate description of the flow force also requires the use of characteristic maps according to eq. (5). In contrary to the previous equation the displacement $x_{M}$ of the valve spool isn't explicitly included any more.

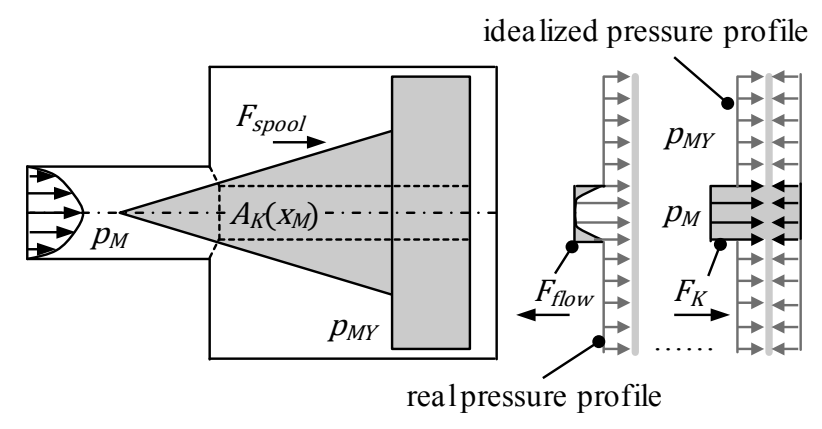

Figure 5: Force on the valve spool

\subsection{Mechanical subsystem}

The valve performance of pressure valves is governed by flow-induced force effects which are in mechanical equilibrium with the solenoid $F_{M}$ and spring force $F_{F}$. The equation of motion (6) summarizes all force effects dependent on valve displacement $x_{M}$, time $t$ and solenoid current $I_{M}$. In general, frictional and damping forces are present, their implementation is simplified and reduced to a viscous friction coefficient $b_{M}$. However, this simplification has no influence on the static valve performance.

$$
m_{M} \ddot{x}_{M}+b_{M} \dot{x}_{M}-F_{K}+F_{\text {flow }}+F_{M}-F_{F}=0
$$

Since the design of the static valve performance is the major focus of this paper all derivatives with respect to time $\partial / \partial t$ are neglected later.

\subsection{Solenoid subsystem}

The solenoid depicted in fig. 6 is an electromechanical transformer. The current $I_{M}$ in the coil causes a magnetic flux in the magnetic circuit consisting of armature, yoke and pole tube. In the residual air gap arises an attractive solenoid force $F_{M}$ between armature and pole tube. Their dependence on the current $I_{M}$ and the armature displacement $\left(X_{M}+X_{M O}\right)$ is determined by the geometrical shapes of the elements in the magnetic circuit as well as their nonlinear material proper- 
ties. The solenoid's force-displacement curves are represented in the following modeling steps by a characteristic map; see also eq. (7). A time dependence of the solenoid's force is neglected because the dynamic performance of typical actuators is sufficient for applications in pressure valves.

$$
F_{M}=f\left(x_{M}+x_{M 0}, I_{M}\right)
$$
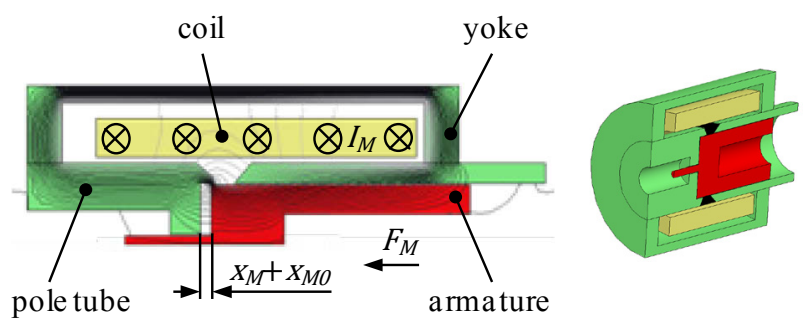

Figure 6: Setup of the electromechanical transformer

\section{Inverse simulation model}

As explained recently the static valve performance is an important property for this valve type. It results from the force interactions of the different physical subsystems outlined in short in fig. (4). System inputs are the flow rate $Q_{M}$ and the solenoid current $I_{M}$, the valve displacement $x_{M}$ and the pressure $p_{A}$ result from the geometrical shape of the involved elements and the other system parameters. Here, the geometrical shape of the control orifice is represented through the loss and geometrical coefficient or the associated characteristic maps. The solenoid's geometry is included by its force-displacement curves.

\subsection{Forward problem formulation}

Starting point for the inverse simulation is the mathematical description of the pressure-flow rate characteristics, because these curves represent the subsequent inputs later. The pressure $p_{A}$ is the sum of three pressure drops eq. (8), whereas the important term is the pressure loss $p_{M}-p_{M Y}$ at the control orifice. The computation of the other pressure losses in dependence of the flow rate is a simple problem.

$$
p_{A}=\Delta p_{A-M}+\Delta p_{M-M Y}+\Delta p_{M Y-Y}+p_{Y}
$$

After combining the equation of motion with the equation for the hydraulic resistance a new transcendent equation (9) establishes the linkage between the valve functionality and the subsystem properties.

$$
Q_{M}^{2}=\frac{F_{M}-F_{F}}{\zeta_{M-M Y} \frac{\rho}{2} \frac{A_{K}}{A_{\text {flow }}{ }^{2}}-\frac{\rho}{A_{\text {flow }}} k_{G F}}
$$

The above equation forms the basis for the following considerations. It defines an implicit relation between the flow rate and the valve displacement. With the knowledge of the flow rate all pressure drops are computable and the static valve performance curves result from the $p_{A}=\mathrm{f}\left(Q_{M}\right)$ linkage. Without the use of the coefficients $\zeta_{M-M Y}$ and $k_{G F}$ another solution strategy is required. Therefore, the flow rate becomes an additional iteration parameter. Now, the equivalent root problem eq. (10) needs to be solved in a proper way. The major difficulty lies in the transcendent character of this equation and that the convergence is evaluated in the pressure drop at the control orifice while the iteration parameter is given through the flow rate. With the help of an iterative problem-solving approach a feasible solution was found.

$$
\begin{aligned}
& \Delta p_{M-M Y}\left(Q_{M}, x_{M}\right) \\
= & \Delta p\left(x_{M}, F_{\text {flow }}\left(Q_{M}, \Delta p_{M-M Y}\right), F_{M}-F_{F}\right)
\end{aligned}
$$

Equations $(9,10)$ illustrate how modifications of the geometrical shape influence the valve performance. This happens through changes in the coefficients or in the characteristic maps for the control orifice and the solenoid.

\subsection{Inverse problem formulation}

Based on the mathematical formulation of the static valve performance an inverse solution strategy is developed. Here, the new inputs for the simulation model are the characteristic curves $p_{A}=\mathrm{f}\left(Q_{M}\right)$ for $I_{M}=$ const. Furthermore, these inputs form the basis for a subsequent solenoid design in combination with the already mentioned predefined control orifice concepts.

The mathematical solution involves several solution steps. First, the hydraulic resistances for the upstream and downstream flow region have to be subtracted from the input curves according to eq. (8) remaining the pressure drop-flow rate relation for the control orifice. The next step is indispensably for the calculation of the force-displacement curves. Here, the essentially needed linkage between pressure drop, flow rate and valve displacement have to be determined. Therefore, the resistance equation is transformed into a root problem eq. (11). Because of the predefined control orifice concepts only the smallest cross-section $A_{\text {flow }}$ is unknown. The geometrical shape of the control orifice directly connects the flow area with the valve displacement. An equivalent solution is possible when the characteristic map $\Delta p=\mathrm{f}\left(Q_{M}, X_{M}\right)$ is used instead of the model equation.

$$
0=\zeta_{M-M Y} \frac{\rho}{2}\left(\frac{Q_{M}}{A_{\text {flow }}\left(x_{M}\right)}\right)^{2}-\left(p_{M}-p_{M Y}\right)
$$

If the valve displacement is known the sum of solenoid and spring force $F_{M}-F_{F}$ can explicitly be computed according to eq. (9). The use of characteristic maps yields to a similar equation.

$$
\begin{gathered}
F_{M}-F_{F}=Q_{M}{ }^{2}\left(\zeta_{M-M Y} \frac{\rho}{2} \frac{A_{K}}{A_{\text {flow }}{ }^{2}}-\frac{\rho}{A_{\text {flow }}} k_{G F}\right) \\
F_{M}-F_{F}=A_{K} \Delta p_{M-M Y}-F_{\text {flow }}\left(Q_{M}, \Delta p_{M-M Y}\right)
\end{gathered}
$$

Equations $(12,13)$ summarize the two different solution opportunities. They offer a possibility to compute the force characteristics for the solenoid-spring combination without any optimization algorithm. The solution strategy bases on the idea that different geometrical control orifice concepts require various counter forces for realization of the intended valve performance. Hence, the choice of a control orifice put requirements on the solenoid's force-displacement characteristics whose achievement by a geometrical design remains 
unanswered so far. The next logical step includes the calculation of the force demands for the different predefined control orifice concepts and their benchmarking with respect to a general feasibility. The following section focuses on these issues.

\section{Geometrical valve design}

\subsection{Control orifice concepts}

As explained in the previous sections every control orifice concept can be described with four characteristic curves. The geometrical values are the closest cross-section $A_{\text {flow }}\left(X_{M}\right)$ and the theoretical pressure area $A_{K}\left(X_{M}\right)$. The energy losses are modeled with the loss coefficient $\zeta_{M-M Y}$ and the force reactions with the geometrical coefficient $k_{G F}$. Alternatively, characteristic maps are used instead of the coefficients describing the fluid flow. For the pressure relief valve three different geometrical concepts were investigated; see fig. 7. These include a valve seat with a chamfer and a valve spool shaped in a cone-/piston-/sphere-like manner. This selection ensures geometric similarity meanwhile all other differences result from the flow-dependent model parameters.

\section{predefined control orifice concepts}
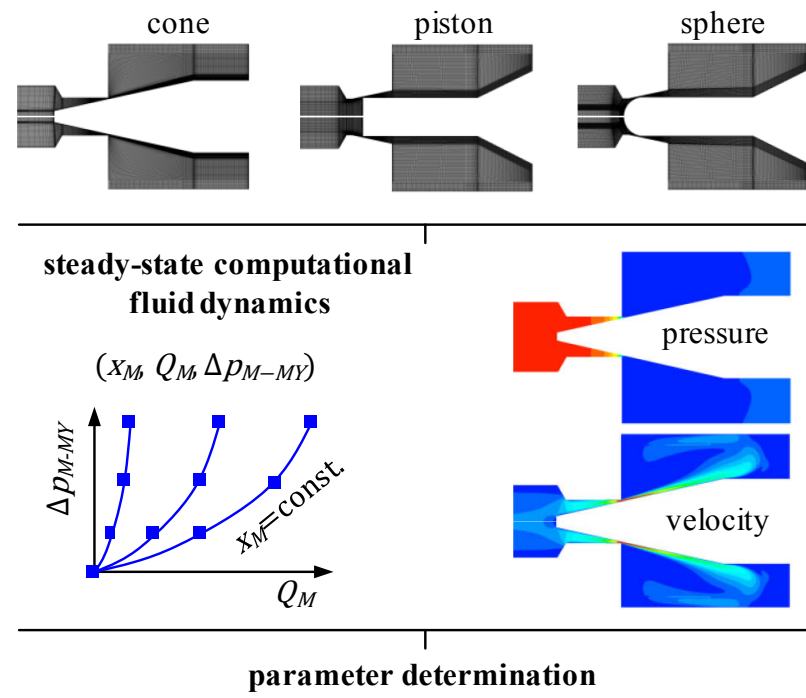

loss/geometrical coefficient

characteristic maps
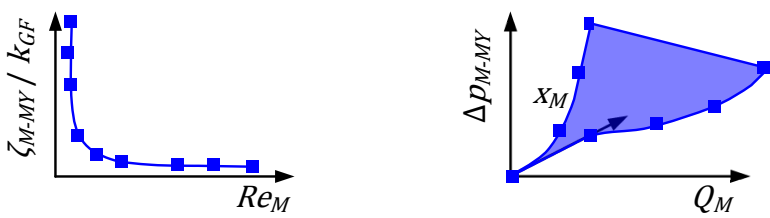

Figure 7: Determination of the hydraulic parameters

The numerical CFD simulations are carried out for several valve displacements analyzing the pressure drop at the control orifice and the force reaction on the valve spool. Assuming similar flow conditions at different valve displacements, a reduction of the obtained results to a 1-dimensional model description is possible. Otherwise, the complex 3-dimensional fluid flow is expressed in terms of characteristic maps.
Figure 8 illustrates the results for the hydraulic resistance and the flow force depending on the Reynolds' number $R e_{M}$.
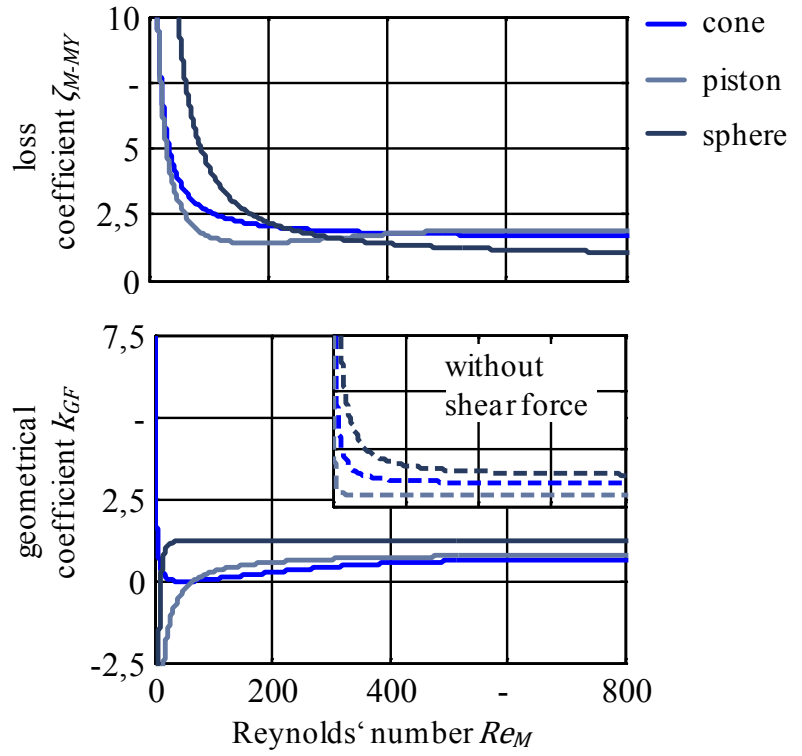

Figure 8: Control orifice characteristic curves

The results of fig. 8 show the different hydraulic behavior of the investigated control orifice concepts. The differences in the laminar region of the loss coefficient $\zeta_{M-M Y}$ can easily be explained through the geometrical shape of the control orifice. Neglecting the shear forces at the valve spool lead to similar characteristic curves for the geometrical coefficient $k_{G F}$. Since the shear forces support the valve opening the acting flow forces are reduced and the geometrical coefficient displays these distinctions. Especially for the coneand sphere-shaped orifice geometry there exist large differences in the remaining flow forces.

\subsection{Determination of counterforce characteristics}

In this subsection, the previously computed characteristic curves for the control orifice concepts are combined with the intended valve performance. For reasons of simplification the whole pressure difference should operate at the control orifice, thus no additional upstream and downstream resistances exist. The results outlined in fig. 9 indicate various differences between the control orifice concepts. For the same static valve performance there exist different demands on the valve displacement. The reason, of course, lies in the varying loss coefficient. In contract to this, the slopes of the force characteristics dominantly result from the hydraulic forces, in particular the flow force. Additionally, the force slopes differ between a low and high pressure setting entailing a spreading dependent on the applied orifice design.

The benchmark of the different orifice concepts leads to a favorable design. As a result, the cone-shaped control orifice remains for the following considerations because of its advantageous counterforce slopes. The decreasing force slope at maximum pressure setting ensures that almost the whole solenoid force is used for closing the valve seat. This supports the development of a smaller actuator. Furthermore, 
the convenient spreading of the force slopes facilitates the realization through a solenoid.
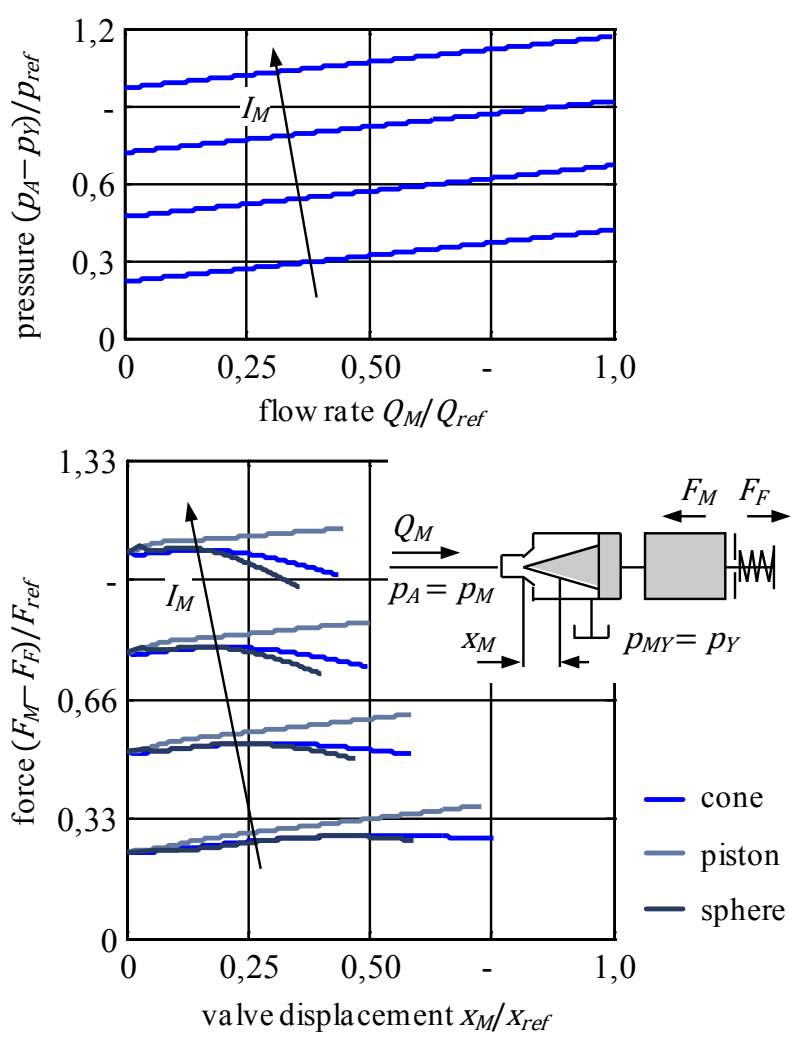

Figure 9: Pressure-flow rate demands and computed counterforce maps

Against the backdrop of a subsequent geometrical solenoid design the required counterforce map contains features of a proportional and switching solenoid. In combination with a spring the realization of this force demands appears possible. How accurate the implementation succeeds is presented in the subsequent section for the solenoid design.

\subsection{Solenoid design}

Starting from the preliminary draft the force requirements have to be fulfilled by a derived solenoid and its geometrical design. As already mentioned the force characteristics combine a switching and proportional behavior. The idea behind the solenoid design is to realize the force spreading inside the actuator and modify the force level with a linear spring. This strategy shows fig. (10). Here, the three governing demands are the maximum force to achieve the highest pressure setting, the necessary operating range the counterforce have to be present and the spreading in the force slopes to obtain a uniform pressure rise independent of the pressure setting. A backward calculation of the solenoid force from the counter forces is nontrivial, because additional parameters characterizing the spring are introduced. These parameters are the spring rate and the spring preload. Furthermore, the used armature displacement range defined through the residual air gap is also of interest. For simplification, the design demands for the solenoid are formulated without the spring force. This allows further adjustments after the geometrical design of the solenoid.

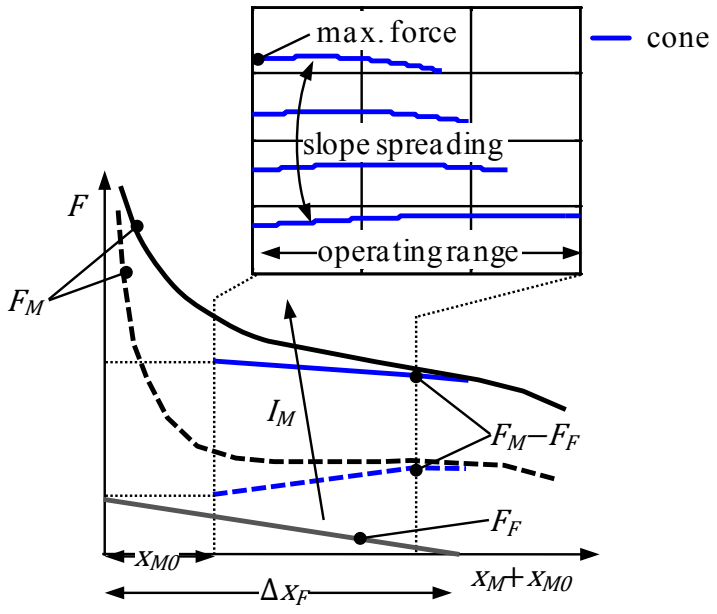

Figure 10: Analysis of the required force characteristics

Based on the requirements the design of the force-influencing air gap in the pole tube was done by means of response surface methodology. Afterwards, different combinations of the solenoid's force characteristics including the spring force were compared until an adequate solution was reached. A final comparison clarifies fig. (11). There, the results agree well at low pressure settings, at higher pressure settings only the average slope is reached. The affect of these differences on the overall valve performance in contract to the preliminary design is discussed in the following sections.

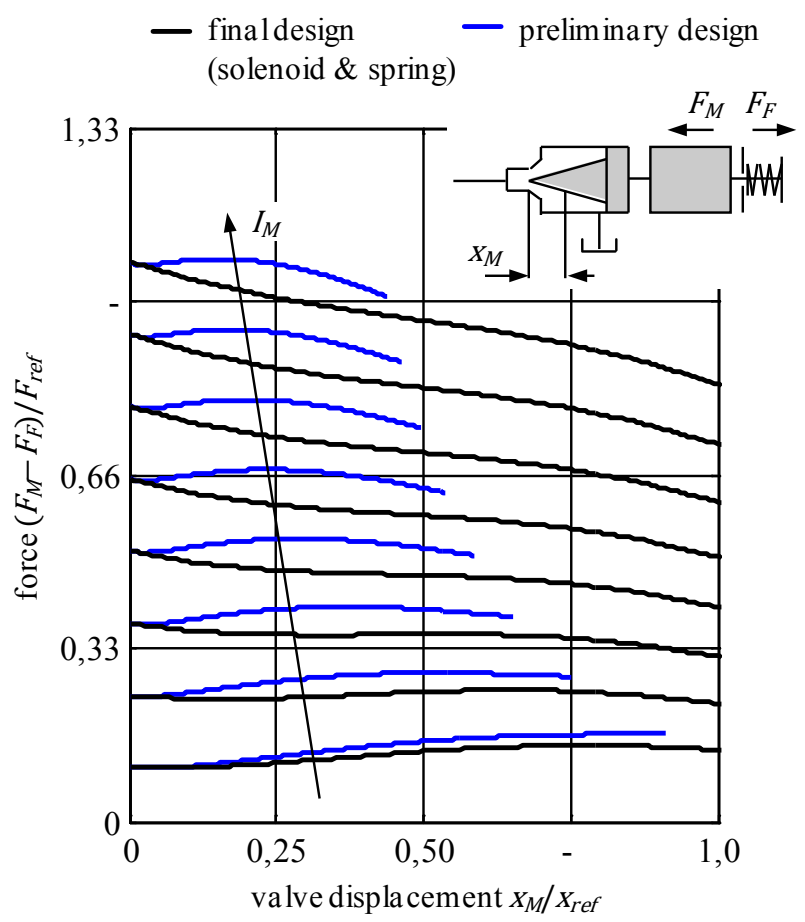

Figure 11: Achieved force characteristics: solenoid-spring combination

In taking a deeper insight into the resulting counterforce map, the force slopes reveal as source of uncertainties. This means, that a deviation of the valve adjustment can cause force errors, even if the compensation of the pressure setting 
through spring preload modifications is generally possible. However, additional errors due to the different reference position may exist. The answer of this question is explained in the second last section.

\section{Measurement and simulation results}

After the fundamental preliminary design of the solenoid on the basis of control orifice concepts with the help of the inverse simulation model the real prototype valve and its subsystem characteristics are considered in a simulation model. Therefore, the already presented solenoid forcedisplacement characteristics are compared with measurement results of the manufactured solenoid. The characteristic curves of the solenoid are measured without the hydraulic elements of the valve assembly. Here, the armature of the solenoid acts against a force transducer, their displacement is measured contactless via laser triangulation meanwhile the coil is set under direct current.

The measurement results in fig. 12 show a good agreement with the simulation at high solenoid currents. At lower magnetic potentials the differences between measurements and simulation rise slowly approving the used simulation methods for a geometrical solenoid design. Anyway, the most influencing factors and crucial simulation parameters in this designing stage are the material properties of the elements in the magnetic circuit. The accuracy of the FEM simulation results is very sensitive to this parameter making intensive foregoing considerations necessary. After the actuator verification the next steps involve the evaluation of the hydraulic subsystem and of the whole component performance.

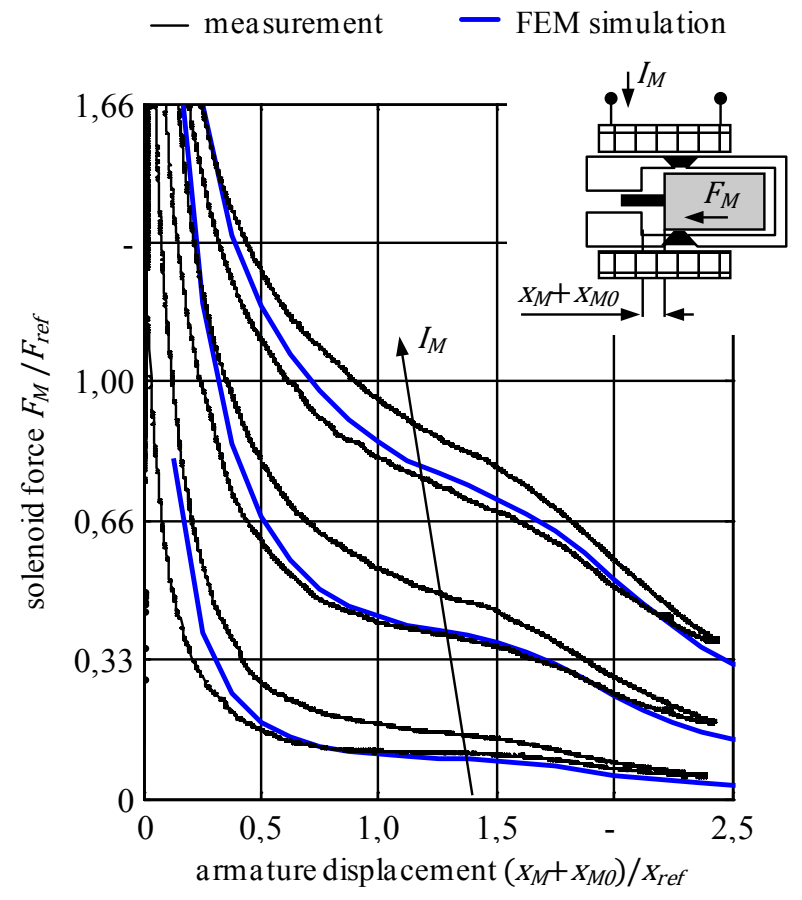

Figure 12: Solenoid force-displacement characteristics: measurement vs. simulation

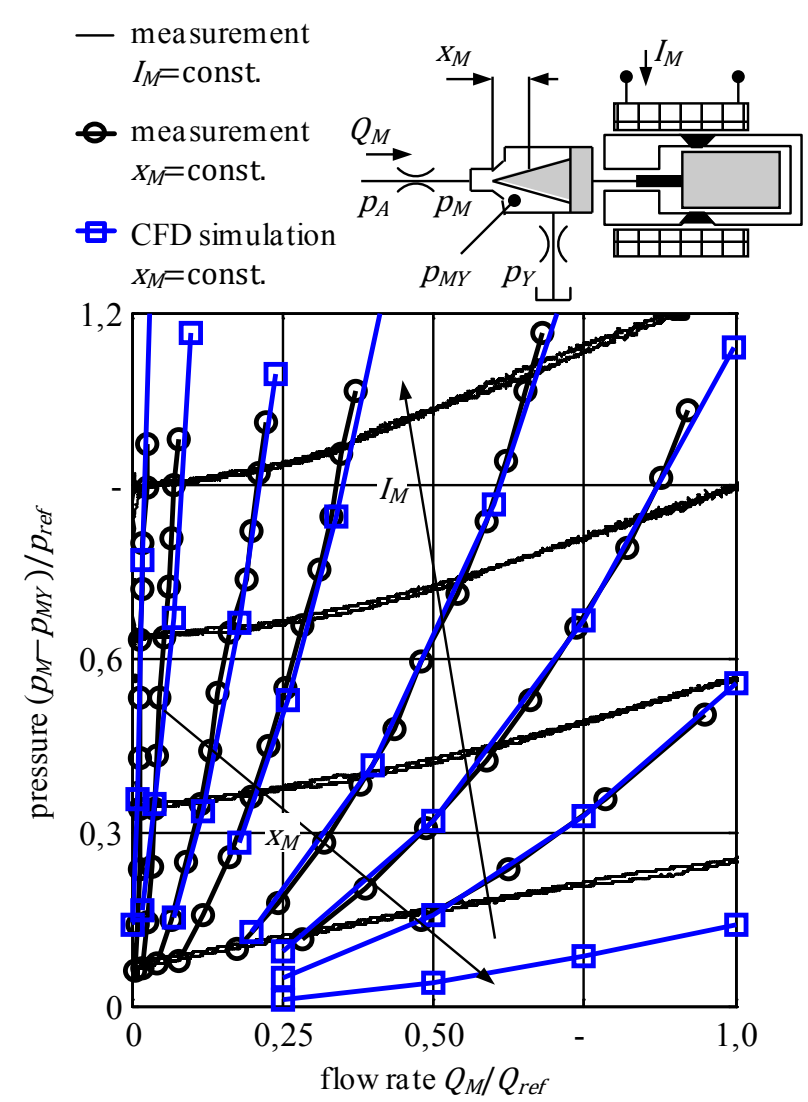

Figure 13: Control orifice pressure drop-flow rate characteristics: measurement vs. simulation

The verification of the hydraulic subsystem is done through the comparison of the pressure drop-flow rate characteristics of the control orifice. The force reaction on the valve spool isn't measured directly, for what reason the validation of the flow forces can only be done indirectly by comparing the valve performance curves. In accordance to the CFD simulation the pressure loss have to be evaluated for a constant valve displacement. Thus, the static valve performance is measured quasi-statically and the desired values are extracted from these results as illustrated in fig. 13 subtracting the upstream and downstream pressure loss before. The measurement of the valve displacements during valve operation is quite complex, because no additional leakage or friction should be incorporated into the system. For this reason, an optic measurement method was chosen and the armature displacement was captured with a laser vibrometer. The presented measurement results are in very good agreement with the simulation results and acknowledge the applied parameter description according to fig. 8. Because no direct force reaction estimation is possible the next step includes comparisons of the whole valve performance.

For verification of the valve performance, there are several issues to take into account, especially the valve adjustment. Here, the valve setting inclusive spring preload has to be identically in measurement and simulation, which requires special efforts in measurement preparation. After this, the static valve performance is validated through the three state variables pressure drop/flow rate and valve displacement. Because the functional specification is given as a function of 
flow rate all characteristic curves in fig. 14 are plotted against the flow rate.
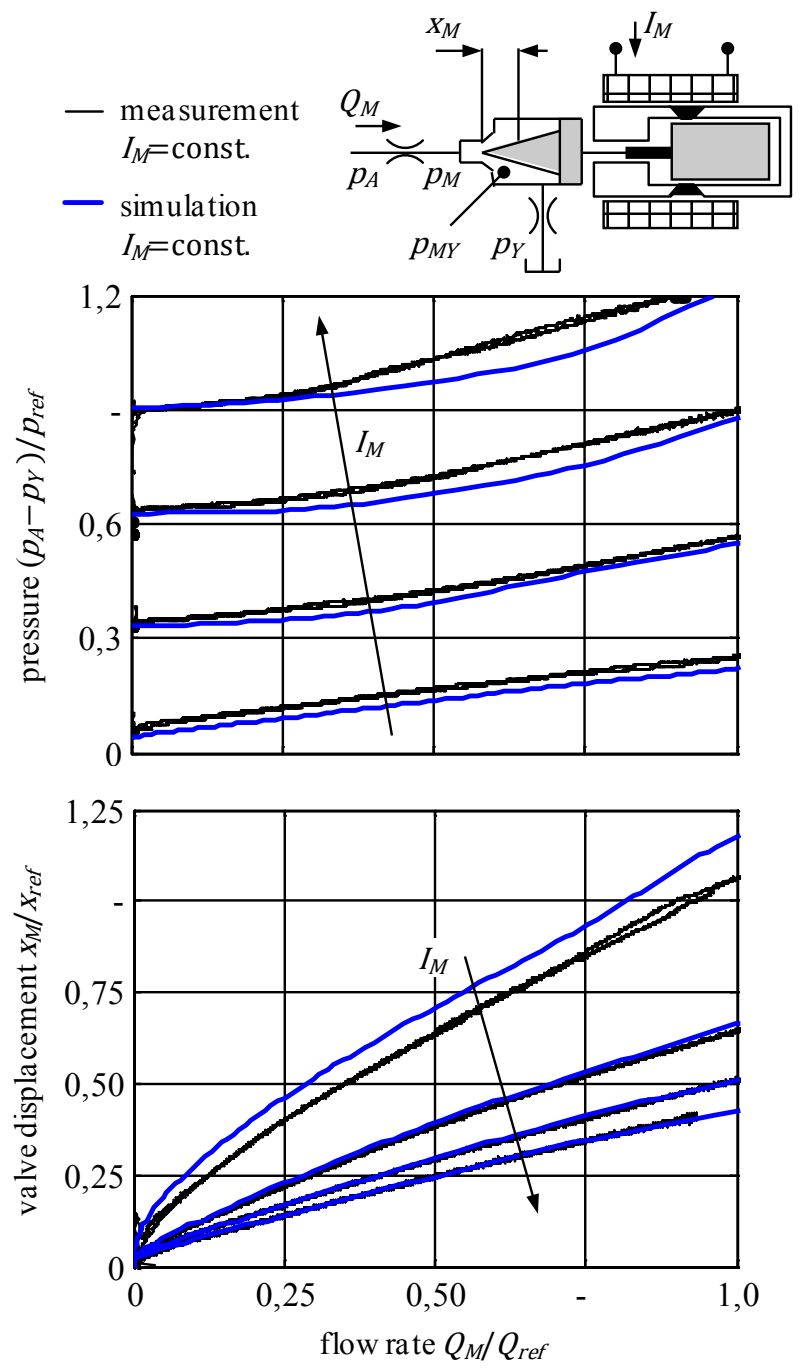

Figure 14: Static valve performance: measurement vs. simulation

The valve displacements in measurement and simulation agree very well with each other. The reason for this was already explained in the verification of the hydraulic subsystem, see also fig. 13. Therefore, the existing differences result only from the slight deviations in the opening pressures. Another situation illustrates the pressure-flow rate characteristic curves. The correlation between measurement and simulation differs increasingly with the opening pressure and the flow rate. A detailed investigation of these deviations inside the CFD simulation shows the formation of a stagnation point within the downstream flow region. This disturbs the force balance at the valve spool and as a consequence the valve performance. At this point, no further investigations are done.

The comparison between the intended and achieved static valve performance confirms the applicability of this simulation strategy for valve design. The differences at low flow rates and high pressure settings can be explained with the counterforce map according to fig. 11 . The additional pres- sure rise at higher flow rates and opening pressures is the result of a stagnation point. Its effect on the force balance can be suppressed through further modifications not discussed in detail here.

Besides the static valve performance pressure relief valves tend to instability requiring additional considerations during the designing stage. The equations for the physical-based description of the valve performance include parameters that only act on the dynamic valve performance. These variables introduce enough degrees of freedom to perform a dynamic valve design in parallel to the static one. No dynamic simulations in the time domain for the whole valve assembly were done, but further measurements to confirm the desired system damping. Figure 15 summarizes some of the measurement results for a nominal current jump at the solenoid at different flow rates.
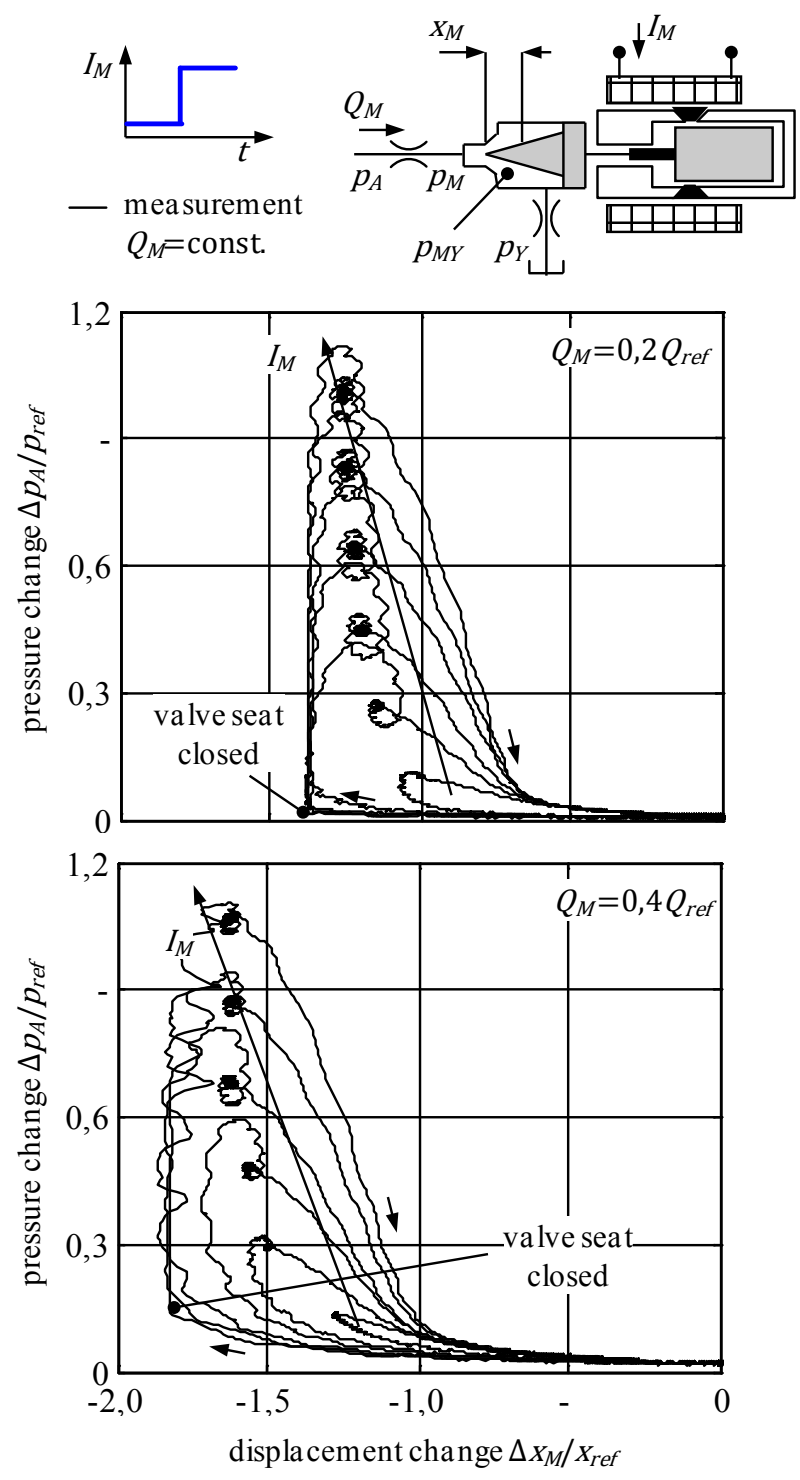

Figure 15: Dynamic valve performance: measurement

The measurements show, that in dependence of the size of the current jump the valve seat is temporarily closed by the solenoid. This tendency decreases with increasing flow rates. Furthermore, the system damping increases with the 
flow rate and decreases with the size of the current jump. However, the system damping is more than sufficient in every operating point sometimes characterized by slight overshoots in the pressure. The primary causes of these overshoots are the small slopes of the pressure-flow rate characteristic curves at higher opening pressures and small flow rates, because the system damping is contrarily to the pressure-flow rate slopes. All in all, the geometrical design of the proportional pressure relief valve provides good results for the overall valve performance without integration of measurements during the virtual design process. Finally, the robustness of this valve assembly is investigated. The reason for this originates from the existing uncertainties within the valve assembly and the related adjustment effort.

\section{Sensitivity and robustness analysis}

The sensitivity and robustness analysis contain of several solution steps outlined in fig. 16. The computation is done with the so called Monte-Carlo simulation. First, a simulation model for the system must be available including the system parameters and their uncertainties. For every random parameter, the variance has to be described in an appropriate manner. After that, random samples of the valve are generated and computed within the simulation model. Finally, the extraction of relevant parameters from a set of random characteristic curves yields information about mean value, distribution and dependences of the output variables. Different evaluation possibilities for the massive set of simulation data exist, whereby histogram plots and first-order and totaleffect sensitivity indices are suitable for general nonlinear systems like the proportional pressure relief valves.

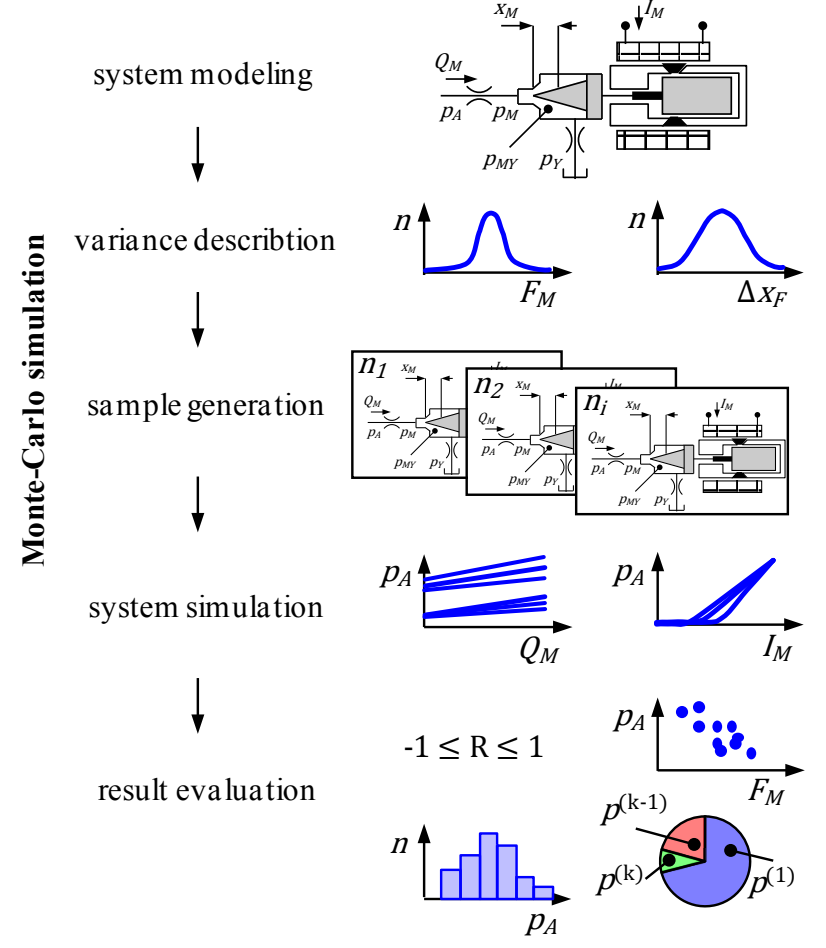

Figure 16: Probabilistic analysis: Monte-Carlo simulation
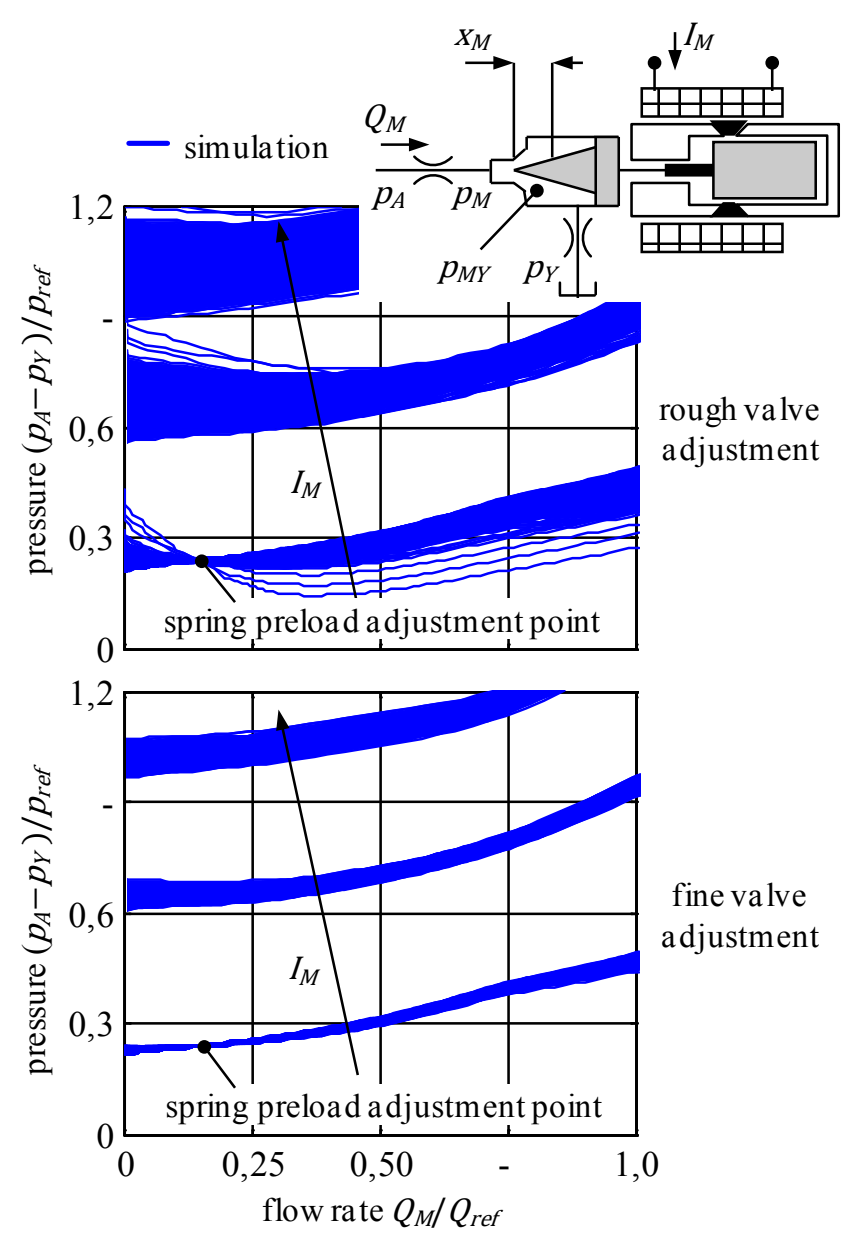

Figure 17: Monte-Carlo simulation: influence of valve adjustment on valve performance

The basis for the Monte-Carlo simulation is the simulation model for the whole valve assembly. Therein, the geometrical tolerances and other uncertainties are integrated. The characteristic curves for the control orifice are held constant during the simulations. In contract to this, force variations of the solenoid are incorporated in a proper way. A major problem during sample generation is the determination of the spring preload because the adjustment point is a simulation point and a priori not known. Assuming a definite valve displacement and flow force for this operating point, the solution of the balance of forces is possible resulting in a distribution for the spring preload. Figure 17 depicts some results of these simulations for two different adjustment accuracies. The results verify the setup procedure for the valve assembly during sample generation. Additionally, the results provide an insight into the massive set of data and the expected pressure distributions.

As illustrated in fig. 17, it would be very helpful to estimate the influence of a single parameter uncertainty onto a particular output distribution. In the linear case, this can be realized with correlation coefficients. Otherwise, the variancebased sensitivity analysis is an appropriate solution strategy. The computation of correlation coefficients eq. (14) is known from basic mathematics. For two random samples $A, B$ the correlation coefficient is in the range of $R=-1$ 
to 1 . No correlation exists if the coefficient is approximately zero; otherwise a partial linear relationship is present. By transferring these basics to the Monte-Carlo simulation correlation coefficients can be calculated for every output variable in relation to every input uncertainty. These values describe, among others, the arrangement of the simulation points in the so called scatter plots.

$$
R(A, B)=\frac{\operatorname{Cov}(A, B)}{\sqrt{\operatorname{Var}(A) \cdot \operatorname{Var}(B)}}
$$

Another possibility is the computation of first-order and total-effect indices using the variance-based sensitivity analysis. Here, the single influences and their interactions with each other are calculated from two sets of independent random samples. For that reason, the two sets of samples have to be computed as well as several resampling matrices of the independent inputs. Together with the necessary sampling length for achieving statistical convergence, computation effort increases intensely. Various formulas published in literature exist for the computation of these indices, whereby the calculation method presented in [11] is used; see also eq. $(15,16)$. Therein, $S_{i}$ denotes the first-order and similarly $S_{T i}$ the total-effect indices.

$$
\begin{gathered}
S_{i}=\frac{\frac{1}{N-1} \sum_{r=1}^{N} f_{B, r} \cdot\left(f_{A_{B}^{i}, r}-f_{A, r}\right)}{\operatorname{Var}\left(f_{A B}\right)} \\
S_{T i}=\frac{\frac{1}{2 N} \sum_{r=1}^{N}\left(f_{A, r}-f_{A_{B}^{i}, r}\right)^{2}}{\operatorname{Var}\left(f_{A B}\right)}
\end{gathered}
$$

Based upon the evaluation basics the simulation results for a proper adjusted valve assembly stated at the bottom of fig. 17 are used for further investigations. Thus, the distribution of the pressure and pressure rise for a constant flow rate is extracted for different solenoid currents and outlined in fig. 18. The flow rate at the spring preload adjustment operating point is chosen as reference for these studies.
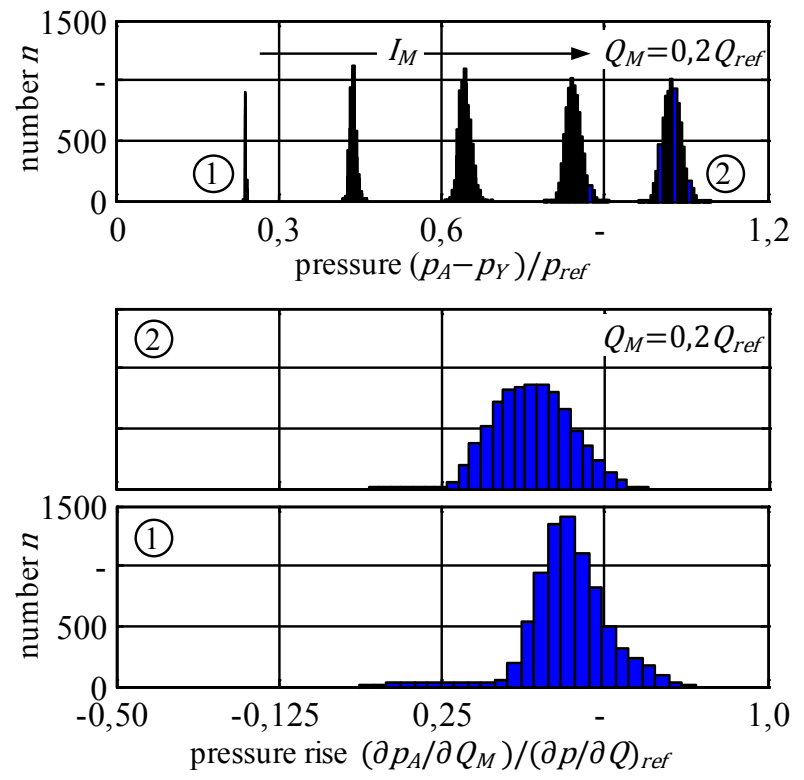

Figure 18: Histogram plots for selected operating points according to fig. 17
Subsequently, the following two issues have to be discussed in detail. In particular, this includes the questions whether the valve performance is robust under the existing uncertainties and to what extend the several influencing parameters contribute to the output variances. This paraphrases in short a robustness and sensitivity analysis. The results indicate an increasing pressure variance with growing pressure setting. This behavior arises predominantly from the valve adjustment and the underlying mounting tolerances of the valve assembly. However, the existing deviations remain within the permissible tolerance. Thus, the designed valve performance fulfills on the one hand the intended mean performance and on the other hand remains rather constant under the influence of parameter uncertainties. This is typically for a robust design generally characterized by a bi-objective optimization problem. The same situation occurs for the pressure rise. The slope variations of the solenoid forcedisplacement curves cause a flattening of the pressure rise at low flow rates. As shown at the top of fig. 17 an insufficient adjustment of the valve can entail decreasing pressure-flow rate curves resulting in instability at low flow rates. This behavior was observed in measurements too. Anyhow, the pressure rise also seems to be robust, additional deviations with higher flow rates arise from flow force differences as a result of the stagnation point influence. In conclusion, the Monte-Carlo simulations confirm some inconsistencies during first measurements and verify the robustness of the valve performance. The next investigations contain a discussion about the composition of the output variations.

The sensitivity analysis is carried out with the help of correlation coefficients and sensitivity indices. The obtained results for the operating points out of fig. 18 are depicted in fig. 19. The correlation coefficients clarify linear tendencies between the inputs and outputs. In contract to the sensitivity indices these results contain directional information expressed by its sign. The pressure variations are primarily determined by the valve adjustment and the solenoid. The effective direction is different between these two influencing parameters that compensation effects are possible. An identical situation exists for the pressure rise only the acting directions are reversed. The other sources of uncertainty seem to have no significant influence on the output variations. One look at the sensitivity indices indicates a similar situation. Because no larger differences between the firstorder and total-effect indices are present the result interpretation is reduced to first-order indices. The equality of these values points towards the absence of interactions between the input parameters in the range of their single uncertainties. Here, the situation is clearly equivalent to the correlation coefficients. The uncertainties of the valve adjustment and of the solenoid affect primarily the output variances. The partial values depend on the pressure setting and are in this parameter combination nearly evenly distributed. As a result, the first-order indices clearly show the principal influential parameters. Further enhancements of the robustness of the valve performance should begin with modifications of these uncertainties. With this, the simulation-based design of the valve performance and its robustness is completed showing the applicability of the acquired and existing simulation methods for an entire and successful virtual valve design. 


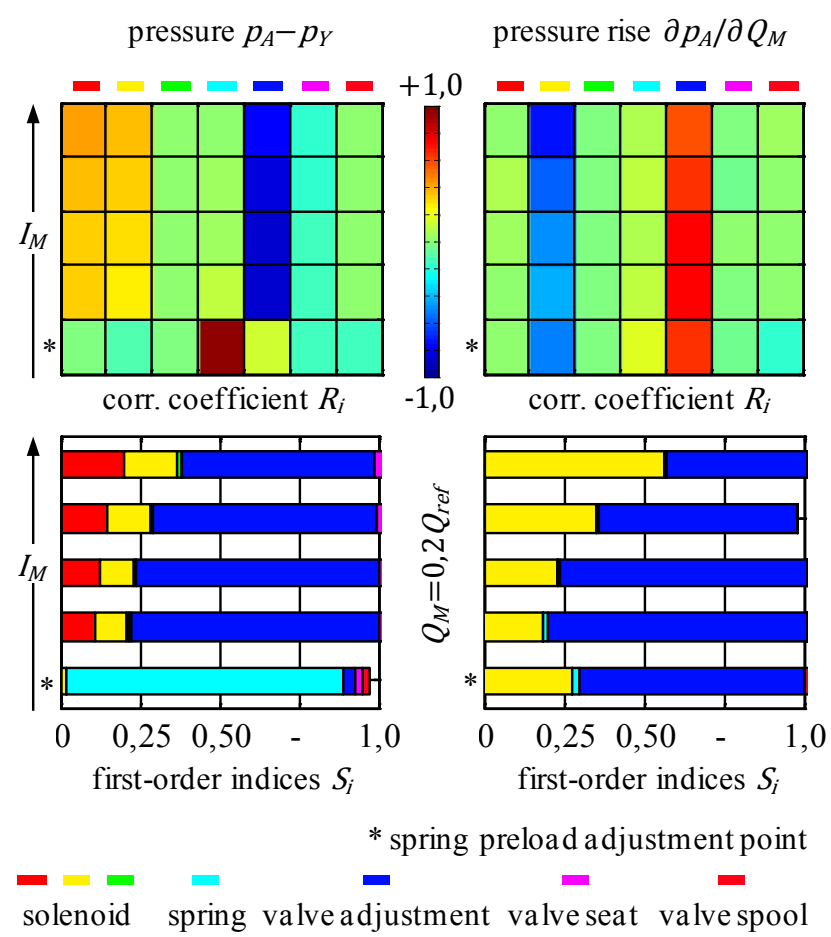

Figure 19: Correlation coefficients and first-order sensitivity indices according to fig. 18

\section{Conclusion and Outlook}

This paper explains an entire solution strategy in virtual valve design starting with a functional specification of the intended valve performance of a proportional pressure relief valve. Based upon these requirements a novel numerical procedure is developed enabling a direct computation of the necessary solenoid force-displacement characteristic curves. Afterwards, the implementation of these requirements is realized through a specifically designed geometrical shape of the solenoid. In parallel, different control orifice concepts are benchmarked resulting in a favorable solution for the whole valve assembly. The virtual designed valve basically consisting of a control orifice and a solenoid is built up and its operability is verified on a test rig. The qualitative and quantitative correlation between measurement and simulation results confirms the chosen simulation strategy and its applicability in virtual product design. Finally, a robustness analysis reinforces the whole valve design regarding uncertainties at the control orifice, the solenoid and the compounded valve assembly. The sensitivity and robustness analyses support the understanding of measurement observations arising from varying valve adjustments as well as the identification of important parameters for further enhancements of the valve operability.

Furthermore, this inverse simulation attempt can be extended to a geometrical design of the control orifice on the subsystem and component level instead of using predefined geometrical concepts. With it, the whole design process of a proportional pressure valve is covered through simulation methods greatly supporting the virtual product developing starting from a functional specification.

\section{Nomenclature}

\begin{tabular}{lll}
\hline Designation & Denotation & Unit \\
\hline$A, B$ & random input variables/matrices & {$[-]$} \\
$A_{K}$ & pressure area & {$\left[\mathrm{mm}^{2}\right]$} \\
$A_{\text {flow }}$ & smallest flow cross-section & {$\left[\mathrm{mm}^{2}\right]$} \\
$b_{M}$ & viscous damping coefficient & {$[\mathrm{m} / \mathrm{s}]$} \\
$f_{A} f_{B}, f_{A B}$ & random output variables/matrices & {$[-]$} \\
$F_{\text {flow }}$ & flow force & {$[\mathrm{N}]$} \\
$F_{F}$ & spring force & {$[\mathrm{N}]$} \\
$F_{K}$ & pressure force & {$[\mathrm{N}]$} \\
$F_{M}$ & solenoid force & {$[\mathrm{N}]$} \\
$I_{M}$ & solenoid current & {$[\mathrm{A}]$} \\
$k_{G F}$ & geometrical coefficient & {$[-]$} \\
$m_{M}$ & mass & {$[\mathrm{g}]$} \\
$n$ & number & {$[-]$} \\
$\Delta p$ & pressure drop/pressure loss & {$[\mathrm{bar}]$} \\
$p_{A}$ & operating pressure & {$[\mathrm{bar}]$} \\
$p_{M}$ & upstream pressure & {$[\mathrm{bar}]$} \\
$p_{M Y}$ & downstream pressure & {$[\mathrm{bar}]$} \\
$p_{Y}$ & tank pressure & {$[\mathrm{bar}]$} \\
$Q_{M}$ & flow rate & {$[1 / \mathrm{min}]$} \\
$R$ & correlation coefficient & {$[-]$} \\
$S_{i}$ & first-order sensitivity index & {$[-]$} \\
$S_{T i}$ & total-effect sensitivity index & {$[-]$} \\
$\Delta X_{F}$ & spring preload & {$[\mathrm{mm}]$} \\
$X_{M}$ & valve displacement & {$[\mathrm{mm}]$} \\
$X_{M O}$ & residual air gap & {$[\mathrm{mm}]$} \\
$\zeta$ & loss coefficient & {$[-]$} \\
$\rho$ & density & {$\left[\mathrm{kg} / \mathrm{m}^{3}\right]$} \\
& & \\
& &
\end{tabular}

\section{References}

[1] G Licskó, A Champneys, and C Hős. Nonlinear Analysis of a Single Stage Pressure Relief Valve. International Journal of Applied Mathematics, 39(4), 2009.

[2] C Bazsó, and C Hős. An experimental, numerical and theoretical study on valve chatter. Proc. of the Fluid Power and Motion Control, Bath, 2010

[3] D Cristofori, and A Vacca. The Modeling of Electrohydraulic Proportional Valves. Journal of Dynamic Systems, Measurement and Control, 134, 2012 
[4] T D Spegar et al. An Analytical and Experimental Study of a High Pressure Single Piston Pump for Gasoline Direct Injection (GDi) Engine Applications. SAE Technical Paper, 2009-01-1504, 2009

[5] J Faulkner, N Johnston, and S Weber. Computer Modeling of a Pressure Relief Cartridge Valve. Proc. of the $52^{\text {nd }}$ National Conference on Fluid Power, Las Vegas, 2011

[6] D Wehner. Modellbasierter Systementwurf am Beispiel vorgesteuerter Druckbegrenzungsventile. Dissertation, Technische Universität Dresden, 2008

[7] M Erhard, D Wehner, and J Weber. Numerische Strömungsberechnung (CFD) im Ventilentwurf. FluidStruktur-Interaktion (FSI) und deren Anwendungspotenzial. Ölhydraulik und Pneumatik, 11-12, 2011

[8] M Petzold et al. Analysis of the flow conditions in a dosing pump with regards to new fuels. Proc. of the $8^{\text {th }}$ International Fluid Power Conference, Dresden, 2012

[9] P Casoli, and A Vacca. Design Optimization of a special relief valve with Response Surface Methodology. Proc. of the Power Transmission and Motion Control, Bath, 2007

[10]M Erhard, G Schoppel, and J Weber. Simulation-Based Design of a Direct-Operated Proportional Pressure Relief Valve. Proc. of the $8^{\text {th }}$ International Fluid Power Conference, Dresden, 2012

[11]S Han. Varianzbasierte Sensitivitätsanalyse als Beitrag zur Bewertung der Zuverlässigkeit adaptronischer Systeme. Dissertation, Technische Universität Darmstadt, 2011 